\title{
Generalized Classical Weighted Means, the Invariance, Complementarity and Convergence of Iterates of the Mean-Type Mappings
}

\author{
Dorota Głazowska@ and Janusz Matkowski
}

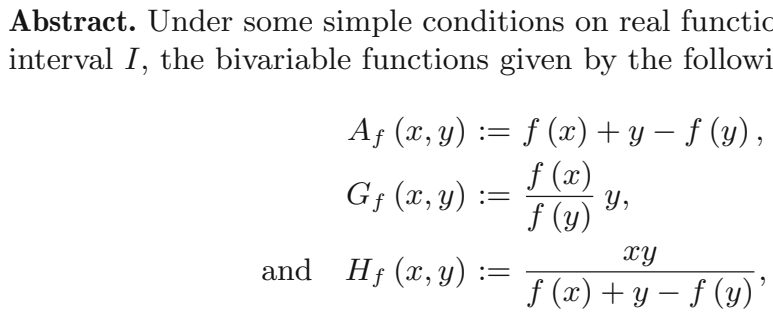

for all $x, y \in I$, generalize, respectively, the classical weighted arithmetic, geometric and harmonic means. The invariance equations

$A_{f} \circ\left(G_{g}, H_{h}\right)=A_{f}, \quad G_{g} \circ\left(A_{f}, H_{h}\right)=G_{g} \quad$ and $\quad H_{h} \circ\left(A_{f}, G_{g}\right)=H_{h}$,

where $f, g, h$ are the unknown functions are, in some special cases, solved. The convergence of iterates of the relevant mean-type mappings is considered. As an application the solutions of some functional equations are determined.

Mathematics Subject Classification. 26E30, 39B12, 39B22.

Keywords. Generalized arithmetic mean, generalized geometric mean, generalized harmonic mean, invariance identity, mean-type mapping, iteration, convergence of iterates, functional equation. 


\section{Introduction}

The classical Pythagorean harmony proportion involving the bivariable symmetric arithmetic mean $\mathcal{A}$, harmonic mean $\mathcal{H}$ and geometric mean $\mathcal{G}$, equivalent to the equality

$$
\mathcal{G} \circ(\mathcal{A}, \mathcal{H})=\mathcal{G}
$$

as well as its extension for the weighted means

$$
\mathcal{G} \circ\left(\mathcal{A}_{t}, \mathcal{H}_{t}\right)=\mathcal{G}
$$

where $t \in(0,1)$, and

$$
\mathcal{A}_{t}(x, y)=t x+(1-t) y, \quad \mathcal{H}_{t}(x, y)=\frac{x y}{t x+(1-t) y}, \quad \mathcal{G}(x, y)=\sqrt{x y}
$$

referred to as the invariance of the geometric mean with respect to the meantype mappings $\left(\mathcal{A}_{t}, \mathcal{H}_{t}\right)$, has well known important consequences. In particular it implies that for every $t \in(0,1)$ the sequence $\left(\left(\mathcal{A}_{t}, \mathcal{H}_{t}\right)^{n}: n \in \mathbb{N}\right)$ of the iterates of the mean-type mapping $\left(\mathcal{A}_{t}, \mathcal{H}_{t}\right)$ converges to $(\mathcal{G}, \mathcal{G})$ (uniformly on compact subsets of $\left.(0, \infty)^{2}\right)[12]$ (also, under stronger conditions, Borwein and Borwein [2]).

This is a special case of the following more general fact. If $M, N$ are continuous bivariable strict means in an interval $I$, then there is a unique mean $K$ invariant with respect to the mean-type mapping $(M, N)$, that is satisfying the identity $K \circ(M, N)=K$; moreover the sequence of iterates $\left((M, N)^{n}: n \in \mathbb{N}\right)$ converges to $(K, K)$ (uniformly on compact subsets of $I^{2}$ ) (see $[9,10,12])$. At this stage the mean $N$ is called complementary to $M$ with respect to $K$ (briefly, a $K$-complementary to $M$ ) and vice versa.

There is a rich literature related to the invariance equation problems. We refer the interested in the results dealing with invariant means, a survey paper [7]. Let us mention that invariance of the arithmetic mean with respect to the quasi-arithmetic mean-type mappings as well as some related questions were considered among others in $[1,4-6,9]$.

Motivated by these facts, we give necessary and sufficient conditions for a real function $f$ defined on an interval $I$, under which the functions $A_{f}, G_{f}$, $H_{f}$ given by the following formulas

$$
\begin{aligned}
& A_{f}(x, y):=f(x)+y-f(y), \\
& G_{f}(x, y):=\frac{f(x)}{f(y)} y, \\
& H_{f}(x, y):=\frac{x y}{f(x)+y-f(y)}
\end{aligned}
$$

for $x, y \in I$, are bivariable means in $I$, generalizing respectively, the weighted arithmetic, geometric and harmonic means. In fact these means are symmetric, if and only if they coincide with $\mathcal{A}, \mathcal{G}, \mathcal{H}$, respectively. The invariance identity

$$
\mathcal{G} \circ\left(A_{f}, H_{f}\right)=\mathcal{G},
$$


extending the Pythagorean harmony proportion and confirming the adequacy of the generalized means, allows to conclude the suitable complementariness of $A_{f}$ and $H_{f}$ with respect to $\mathcal{G}$, and determine the convergence of sequence of the iterates of the mean-type mapping $\left(A_{f}, H_{f}\right)$ to $(\mathcal{G}, \mathcal{G})($ Sect. 2$)$.

In Sect. 3 we consider three related functional equations

$$
A_{f} \circ\left(G_{g}, H_{h}\right)=A_{f}, \quad H_{h} \circ\left(A_{f}, G_{g}\right)=H_{h}, \quad G_{g} \circ\left(A_{f}, H_{h}\right)=G_{g},
$$

where $f, g, h$ are the unknown functions. We solve the first equation in the case when $A_{f}=\mathcal{A}$, the second in the case when $H_{h}=\mathcal{H}$, and the third in the case when $G_{g}=\mathcal{G}$. Moreover, for each of the classical symmetric means $\mathcal{A}, \mathcal{H}, \mathcal{G}$ and for some of the above generalized means $A_{f}, G_{f}, H_{f}$ we prove the existence and uniqueness of the respective complementary mean, we give its explicit formula, as well as the limit of the sequence of iterates of the relevant mean-type mappings.

In the last section we establish the form of all functions which are invariant with respect to the corresponding mean-type mappings and continuous on the diagonal.

\section{Basic Notions and Generalization of the Weighted Arithmetic, Geometric and Harmonic Means}

Let $I \subset \mathbb{R}$ be an interval. A bivariable function $M: I^{2} \rightarrow \mathbb{R}$ is called a mean in $I$, if

$$
\min (x, y) \leq M(x, y) \leq \max (x, y), \quad x, y \in I .
$$

A mean $M$ is called strict if for all $x, y \in I, x \neq y$, these inequalities are sharp, and it is called symmetric, if $M(x, y)=M(y, x)$ for all $x, y \in I$ (see $[2,3])$.

Remark 1. If $M: I^{2} \rightarrow I$ is a mean, then $M(J \times J)=J$ for any subinterval $J \subset I$.

Let $K, M, N: I^{2} \rightarrow I$ be means. If

$$
K(M(x, y), N(x, y))=K(x, y), \quad x, y \in I,
$$

we write briefly $K \circ(M, N)=K$ and we say that:

(a) $K$ is invariant with respect to the mean-type mapping $(M, N): I^{2} \rightarrow I^{2}$, briefly, $K$ is $(M, N)$-invariant;

(b) $N$ is complementary to $M$ with respect to $K$, briefly, $N$ is a $K$ complementary to $M$.

Let us quote the following (see Remark 1 in [9])

Lemma 1. Let $I \subset \mathbb{R}$ be an interval and $K: I^{2} \rightarrow I$ be a symmetric mean which is continuous and strictly increasing with respect to the first variable. Then for every mean $M: I^{2} \rightarrow I$ there exists a unique $K$-complementary mean $N: I^{2} \rightarrow I$. 
Remark 2 ([11]). Let $I \subset \mathbb{R}$ be an interval and let $f, \varphi: I \rightarrow \mathbb{R}$. Then the function

$$
M(x, y)=f(x)+\varphi(y), \quad x, y \in I,
$$

is a mean if and only if $\varphi=\left.\mathrm{id}\right|_{I}-f$, i.e. $M=A_{f}$, where $A_{f}: I^{2} \rightarrow \mathbb{R}$ is defined by

$$
A_{f}(x, y):=f(x)+y-f(y), \quad x, y \in I,
$$

and the functions $f$ and $\left.\mathrm{id}\right|_{I}-f$ are increasing. Moreover

(i) $A_{f}$ is a mean if and only if the function $f$ is increasing and nonexpansive;

(ii) $A_{f}$ is a strict mean if and only if $f$ and id $\left.\right|_{I}-f$ are strictly increasing, or equivalently, if and only if $f$ is strictly increasing and strictly contractive, i.e.

$$
|f(x)-f(y)|<|x-y|, \quad x, y \in I, \quad x \neq y ;
$$

(iii) $A_{f}$ is a weighted arithmetic mean of the weight $t \in[0,1]$, i.e.

$$
A_{f}(x, y)=t x+(1-t) y, \quad x, y \in I,
$$

if and only if the function $I \ni x \longmapsto f(x)-t x$ is constant;

(iv) $A_{f}$ is symmetric if and only if $A_{f}=\mathcal{A}$, or equivalently, if and only if the function $I \ni x \longmapsto f(x)-\frac{x}{2}$ is constant.

Under the assumptions of this remark, the mean $A_{f}$ given by formula (1) is called a generalized weighted arithmetic mean and the function $f$, called its generator, being Lipschitzian, is absolutely continuous; consequently, it is differentiable almost everywhere.

Remark $3([8])$. Let $I \subset(0, \infty)$ be an interval and let $f, \psi: I \rightarrow(0, \infty)$. Then the function

$$
M(x, y)=f(x) \psi(y), \quad x, y \in I,
$$

is a mean if and only if $\psi=\frac{\text { id }\left.\right|_{I}}{f}$, i.e. $M=G_{f}$, where $G_{f}: I^{2} \rightarrow(0, \infty)$ is defined by

$$
G_{f}(x, y):=\frac{f(x)}{f(y)} y, \quad x, y \in I,
$$

and the functions $f$ and $\frac{\left.\mathrm{id}\right|_{I}}{f}$ are increasing. Moreover

(i) $G_{f}$ is a mean if and only if the function $f$ is increasing and

$$
1 \leq \frac{f(y)}{f(x)} \leq \frac{y}{x}, \quad x, y \in I, x<y
$$


(ii) $G_{f}$ is a strict mean if and only if the functions $f$ and $\frac{\left.\mathrm{id}\right|_{I}}{f}$ are strictly increasing, or equivalently, if and only if

$$
1<\frac{f(y)}{f(x)}<\frac{y}{x}, \quad x, y \in I, x<y
$$

(iii) $G_{f}$ is a weighted geometric mean of the weight $t \in[0,1]$, i.e.

$$
G_{f}(x, y)=x^{t} y^{1-t}, \quad x, y \in I,
$$

if and only if the function $I \ni x \longmapsto \frac{f(x)}{x^{t}}$ is a constant;

(iv) $G_{f}$ is symmetric if and only if $G_{f}=\mathcal{G}$, or equivalently, if and only if the function $I \ni x \longmapsto \frac{f(x)}{\sqrt{x}}$ is constant.

Under the assumptions of this remark, the mean $G_{f}$ given by formula (2) is called a generalized weighted geometric mean and the function $f$, called its generator, is absolutely continuous; consequently, differentiable almost everywhere.

Note the following easy to prove

Remark 4. Let $I \subset(0, \infty)$ be an interval and let $f: I \rightarrow \mathbb{R}$. Then the function $H_{f}: I^{2} \rightarrow \mathbb{R}$

$$
H_{f}(x, y)=\frac{x y}{f(x)+y-f(y)}, \quad x, y \in I,
$$

is a correctly defined (strict) mean in $I$ if and only if the functions $f$ and $\left.\mathrm{id}\right|_{I}-f$ are (strictly) increasing. Moreover

(i) $H_{f}$ is a mean if and only if $A_{f}$ is a mean;

(ii) $H_{f}$ is a weighted harmonic mean of the weight $t \in[0,1]$, i.e.

$$
H_{f}(x, y)=\frac{x y}{t x+(1-t) y}, \quad x, y \in I,
$$

if and only if the function $I \ni x \longmapsto f(x)-t x$ is constant;

(iii) $H_{f}$ is symmetric if and only if $H_{f}=\mathcal{H}$, or equivalently, if and only if the function $I \ni x \longmapsto f(x)-\frac{x}{2}$ is constant.

Under the assumptions of this remark, the mean $H_{f}$ given by formula (3) is called a generalized weighted harmonic mean and the function $f$, called its generator, is absolutely continuous; consequently, is differentiable almost everywhere.

The following result shows that, in particular, the above proposed definitions of generalizations of the classical means are natural.

Theorem 1. Let $I \subset(0, \infty)$ be an interval and let $f: I \rightarrow \mathbb{R}$ and $h: I \rightarrow \mathbb{R}$ be such that $f, h,\left.\mathrm{id}\right|_{I}-f$, id $\left.\right|_{I}-h$ are increasing. Then the following conditions are pairwise equivalent

(i) the means $A_{f}$ and $H_{h}$ are mutually complementary with respect to the geometric mean $\mathcal{G}$ (see [9]); 
(ii) the geometric mean $\mathcal{G}$ is invariant with respect to the mean-type mapping $\left(A_{f}, H_{h}\right): I^{2} \rightarrow I^{2}$, i.e.

$$
\mathcal{G} \circ\left(A_{f}, H_{h}\right)=\mathcal{G}
$$

(iii) the function $h-f$ is constant, and

$$
H_{h}=H_{f} .
$$

Moreover, if the functions $f, h$, id $\left.\right|_{I}-f$, id $\left.\right|_{I}-h$ are strictly increasing and $h-f$ is constant, then the sequence of iterates $\left(\left(A_{f}, H_{h}\right)^{n}: n \in \mathbb{N}\right)$ of the mean-type mapping $\left(A_{f}, H_{h}\right)$ converges, uniformly on compact subsets of $I^{2}$, to the mean-type mapping $(\mathcal{G}, \mathcal{G})$.

Proof. Conditions (i) and (ii) are equivalent (see [9]).

Assume (ii). From the definitions of $\mathcal{G}, A_{f}$ and $H_{h}$ (see (1) and (3)), equality (4) holds, if and only if, for arbitrary $x, y \in I$,

$\sqrt{(f(x)+y-f(y)) \frac{x y}{h(x)+y-h(y)}}=\mathcal{G} \circ\left(A_{f}, H_{h}\right)(x, y)=\mathcal{G}(x, y)=\sqrt{x y}$,

which (after simple calculations) can be written equivalently in the form

$$
h(y)-f(y)=h(x)-f(x), \quad x, y \in I .
$$

The above equality holds if and only if the function $h-f$ is a constant, i.e. if and only if there is $c \in \mathbb{R}$ such that

$$
h(x)=f(x)+c, \quad x \in I .
$$

From (3) it follows that $H_{h}=H_{f}$.

To prove the "moreover" result note that, in view of Remark 2 (ii), the function $f$ is continuous and, consequently, the mean-type mapping $\left(A_{f}, H_{f}\right)$ is continuous. Since the coordinate means are strict, the result follows from the main result of [10] (see also [12]).

\section{Invariant Means and Some Open Problems}

In this section we consider some invariance equations involving the introduced generalized weighted means $A_{f}, G_{f}$ and $H_{f}$.

We begin with

Problem 1. Let $I \subset(0, \infty)$ be an interval. Find all functions $f, g, h: I \rightarrow$ $(0, \infty)$ satisfying the equation

$$
A_{f} \circ\left(G_{g}, H_{h}\right)=A_{f},
$$

assuming that $f, g, h$ are, respectively, the generators of generalized weighted arithmetic, geometric and harmonic means.

In the case when $A_{f}$ is symmetric, i.e. if $A_{f}=\mathcal{A}$, we prove the following 
Theorem 2. Let $I \subset(0, \infty)$ be an interval. Assume that $g: I \rightarrow(0, \infty)$ is strictly increasing and the functions $\frac{\left.\mathrm{id}\right|_{I}}{g}, h: I \rightarrow(0, \infty)$ and $\left.\mathrm{id}\right|_{I}-h$ are increasing. If

$$
\mathcal{A} \circ\left(G_{g}, H_{h}\right)=\mathcal{A},
$$

then there exist $a \in(0, \infty)$ and $b \in \mathbb{R}$ such that

$$
g(x)=a x, \quad h(x)=x+b, \quad x \in I,
$$

and

$$
G_{g}(x, y)=x, \quad H_{h}(x, y)=y, \quad x, y \in I .
$$

Proof. According to the definitions of the involved means (see (2) and (3)), Eq. (5) reduces to

$$
\frac{g(x)}{g(y)} y+\frac{x y}{h(x)+y-h(y)}=x+y, \quad x, y \in I,
$$

which, after simple calculations, implies that

$$
y^{2}(g(x)-g(y))=((x+y) g(y)-y g(x))(h(x)-h(y)), \quad x, y \in I .
$$

The assumptions of $g$ and $h$ imply that they are absolutely continuous. Let $x \in I$ be a differentiability point of $g$ and $h$. Dividing both sides of the Eq. (6) by $x-y$, we get

$$
y^{2} \frac{g(x)-g(y)}{x-y}=((x+y) g(y)-y g(x)) \frac{h(x)-h(y)}{x-y}, \quad y \in I, y \neq x .
$$

It follows that $g$ and $h$ are differentiable at the point $x$ and, letting $y \rightarrow x$, gives

$$
x^{2} g^{\prime}(x)=x g(x) h^{\prime}(x), \quad x \in I,
$$

whence

$$
h^{\prime}(x)=\frac{g^{\prime}(x)}{g(x)} x, \quad x \in I .
$$

Differentiating both sides of (6) with respect to the variable $x$ at the point $x$, we obtain

$$
\begin{aligned}
y^{2} g^{\prime}(x)= & \left(g(y)-y g^{\prime}(x)\right)(h(x)-h(y)) \\
& +((x+y) g(y)-y g(x)) h^{\prime}(x), \quad y \in I .
\end{aligned}
$$

On the other hand, differentiating both sides of this equality with respect to $y$ (at the points of differentiability of $g$ and $h$ ), we get

$$
\begin{aligned}
2 y g^{\prime}(x)= & \left(g^{\prime}(y)-g^{\prime}(x)\right)(h(x)-h(y))-\left(g(y)-y g^{\prime}(x)\right) h^{\prime}(y) \\
& +\left(g(y)+(x+y) g^{\prime}(y)-g(x)\right) h^{\prime}(x),
\end{aligned}
$$

and this equality holds true for almost all $x$ and almost all $y$ in $I$. Setting here $y=x$ we have

$$
2 x g^{\prime}(x)=\left(3 x g^{\prime}(x)-g(x)\right) h^{\prime}(x), \quad \text { a.e. in } I,
$$


whence, in view of (7), we obtain

$$
2 x g^{\prime}(x)=\left(3 x g^{\prime}(x)-g(x)\right) \frac{x g^{\prime}(x)}{g(x)}, \quad \text { a.e. in } I,
$$

which simplifies to

$$
g^{\prime}(x)\left(g(x)-x g^{\prime}(x)\right)=0, \quad \text { a.e. in } I .
$$

The absolute continuity and strict monotonicity of $g$ imply that $g^{\prime}(x)>0$ a.e. in $I$, so

$$
g^{\prime}(x)=\frac{g(x)}{x} \quad \text { a.e. in } I .
$$

Since the derivative of the absolutely continuous function $g$ coincides a.e. in $I$ with the continuous function $x \longmapsto \frac{g(x)}{x}$, it follows that $g$ must be continuously differentiable in $I$. Thus $g$ satisfies the differential equation

$$
g^{\prime}(x)=\frac{g(x)}{x}, \quad x \in I .
$$

Solving this equation we get

$$
g(x)=a x, \quad x \in I,
$$

for some $a>0$. Hence, in view of $(7)$ we have

$$
h^{\prime}(x)=\frac{x g^{\prime}(x)}{g(x)}=1, \quad x \in I,
$$

SO

$$
h(x)=x+b, \quad x \in I,
$$

for some real $b$. Hence, by the definitions of $G_{g}$ and $H_{h}$, we get

$$
G_{g}(x, y)=x, \quad H_{h}(x, y)=y, \quad x, y \in I .
$$

Let us note that Lemma 1 and main results of [10] (also [12]) allow to conclude:

Remark 5. Let $I \subset(0, \infty)$ be an interval and let $f: I \rightarrow(0, \infty)$ and $\frac{\left.\mathrm{id}\right|_{I}}{f}$ be increasing functions. Then

(i) there exists a unique function $M: I^{2} \rightarrow \mathbb{R}$ such that

$$
\mathcal{A} \circ\left(G_{f}, M\right)=\mathcal{A},
$$

moreover $M$ is an $\mathcal{A}$-complementary mean for $G_{f}$, and

$$
M(x, y)=x+y-\frac{f(x)}{f(y)} y, \quad x, y \in I ;
$$

(ii) if the functions $f$ and $\frac{\left.\mathrm{id}\right|_{I}}{f}$ are strictly increasing, then the sequence of iterates $\left(\left(G_{f}, M\right)^{n}: n \in \mathbb{N}\right)$ converges to $(\mathcal{A}, \mathcal{A})$ (uniformly on compact subsets of $\left.I^{2}\right)$. 
We can also consider the invariance of the arithmetic mean $\mathcal{A}$ with respect to the mean-type mappings involving at least one of the introduced means $A_{f}$ or $H_{f}$. Similarly as in the above remark we will get the explicit formulas for the respective mean-type mappings ensuring the invariance, the limit of sequence of its iterates, as well as the complementary means, but we omit statements of these results.

Corollary 1. Let $I \subset(0, \infty)$ be an interval. Assume that $f: I \rightarrow(0, \infty)$ and $\frac{\left.\mathrm{id}\right|_{I}}{f}$ are strictly increasing. The $\mathcal{A}$-complementary mean $M: I^{2} \rightarrow I$ for $G_{f}$ obtained in Remark 5 is not a generalized weighted harmonic mean.

Similarly one can raise

Problem 2. Let $I \subset(0, \infty)$ be an interval. Find all functions $f, g, h: I \rightarrow$ $(0, \infty)$ satisfying the equation

$$
H_{h} \circ\left(A_{f}, G_{g}\right)=H_{h},
$$

assuming that $f, g, h$ are, respectively, the generators of generalized weighted arithmetic, geometric and harmonic means.

In the case when $H_{h}$ is symmetric, i.e. if $H_{h}=\mathcal{H}$, we prove the following

Theorem 3. Let $I \subset(0, \infty)$ be an interval. Assume that $f: I \rightarrow(0, \infty)$, $\left.\mathrm{id}\right|_{I}-f, g: I \rightarrow(0, \infty), \frac{\left.\mathrm{id}\right|_{I}}{g}$ are increasing. If

$$
\mathcal{H} \circ\left(A_{f}, G_{g}\right)=\mathcal{H}
$$

then there exist $a \in(0, \infty)$ and $b \in \mathbb{R}$ such that either

$$
f(x)=b, \quad g(x)=a x, \quad x \in I,
$$

and

$$
A_{f}(x, y)=y, \quad G_{g}(x, y)=x, \quad x, y \in I
$$

or

$$
f(x)=x+b, \quad g(x)=a, \quad x \in I,
$$

and

$$
A_{f}(x, y)=x, \quad G_{g}(x, y)=y, \quad x, y \in I .
$$

Proof. For the same reason as in the previous proof, the functions $f$ and $g$ are differentiable in $I$. By the definitions of the $\mathcal{H}, A_{f}$ and $G_{g}$ (see (1) and (2)), Eq. (8) can be written in the form

$$
\frac{2(f(x)+y-f(y)) \frac{g(x)}{g(y)} y}{f(x)+y-f(y)+\frac{g(x)}{g(y)} y}=\frac{2 x y}{x+y}, \quad x, y \in I,
$$


which, after simple calculations, reduces to

$$
((x+y) g(x)-x g(y))(f(x)-f(y))=x y^{2}\left(\frac{g(y)}{y}-\frac{g(x)}{x}\right), \quad x, y \in I .
$$

Dividing both sides of this equation by $x-y$ we have

$((x+y) g(x)-x g(y)) \frac{f(x)-f(y)}{x-y}=-x y^{2} \frac{\frac{g(x)}{x}-\frac{g(y)}{y}}{x-y}, \quad x, y \in I, x \neq y$,

and letting $y$ tend to $x$ we get

$$
x g(x) f^{\prime}(x)=-x^{3} \frac{g^{\prime}(x) x-g(x)}{x^{2}}, \quad x \in I,
$$

whence

$$
f^{\prime}(x)=\frac{g(x)-g^{\prime}(x) x}{g(x)}, \quad x \in I .
$$

Differentiating both sides of (9) in $x$, (after a simplification) we obtain, for all $x, y \in I$,

$$
\begin{aligned}
& \left(g(x)+(x+y) g^{\prime}(x)-g(y)\right)(f(x)-f(y))+((x+y) g(x)-x g(y)) f^{\prime}(x) \\
& \quad=y g(y)-y^{2} g^{\prime}(x) .
\end{aligned}
$$

Now, differentiating both sides of this equality in $y$, we get, for all $x, y \in I$,

$$
\begin{aligned}
& \left(g^{\prime}(x)-g^{\prime}(y)\right)(f(x)-f(y))+\left(g(x)-(x+y) g^{\prime}(x)-g(y)\right) f^{\prime}(y) \\
& +\left(g(x)-x g^{\prime}(y)\right) f^{\prime}(x)=g(y)+y g^{\prime}(y)-2 y g^{\prime}(x) .
\end{aligned}
$$

Taking here $y=x$ we obtain

$$
\left(g(x)-3 x g^{\prime}(x)\right) f^{\prime}(x)=g(x)-x g^{\prime}(x), \quad x \in I .
$$

Note that

$$
g(x)-3 x g^{\prime}(x) \neq 0, \quad x \in I,
$$

as, if $g(x)-3 x g^{\prime}(x)=0$ then also $g(x)-x g^{\prime}(x)=0$, and we would have $g(x)=0$, contradicting the assumption. Thus

$$
f^{\prime}(x)=\frac{g(x)-x g^{\prime}(x)}{g(x)-3 x g^{\prime}(x)}, \quad x \in I .
$$

Hence, making use of (10), we get

$$
\frac{g(x)-g^{\prime}(x) x}{g(x)}=\frac{g(x)-x g^{\prime}(x)}{g(x)-3 x g^{\prime}(x)}, \quad x \in I,
$$

which implies that for every $x \in I$,

$$
\text { either } \quad g(x)-x g^{\prime}(x)=0 \quad \text { or } \quad g(x)=g(x)-3 x g^{\prime}(x) \text {, }
$$

that is, for every $x \in I$,

$$
\text { either } \quad g(x)-x g^{\prime}(x)=0 \quad \text { or } \quad g^{\prime}(x)=0 .
$$


It implies that either there is a constant $a>0$ such that

$$
g(x)=a x, \quad x \in I,
$$

or there is $a>0$ such that

$$
g(x)=a, \quad x \in I .
$$

From (10), in the first case we get $f^{\prime}=0$ in $I$, so there exists $b \in \mathbb{R}$ such that

$$
f(x)=b, \quad x \in I,
$$

and in the second case, $f^{\prime}=1$ in $I$, so, for some real $b$,

$$
f(x)=x+b, \quad x \in I .
$$

Consequently, in the first case we get

$$
A_{f}(x, y)=y, \quad G_{g}(x, y)=x, \quad x, y \in I,
$$

and in the second case,

$$
A_{f}(x, y)=x, \quad G_{g}(x, y)=y, \quad x, y \in I .
$$

Let us mention here also a result, related to the invariance of the harmonic mean, that gives us the explicit formula for an $\mathcal{H}$-complementary mean to generalized weighted arithmetic mean $A_{f}$. The result reads as follows

Remark 6. Let $I \subset(0, \infty)$ be an interval and let $f: I \rightarrow(0, \infty)$ and $\mathrm{id}_{I}-f$ be increasing functions. Then

(i) there exists a unique function $M: I^{2} \rightarrow \mathbb{R}$ such that

$$
\mathcal{H} \circ\left(A_{f}, M\right)=\mathcal{H}
$$

moreover $M$ is an $\mathcal{H}$-complementary mean for $A_{f}$, and

$$
M(x, y)=\frac{x y(f(x)+y-f(y))}{(x+y)(f(x)-f(y))+y^{2}}, \quad x, y \in I ;
$$

(ii) if the functions $f$ and id $\left.\right|_{I}-f$ are strictly increasing, then the sequence of iterates $\left(\left(A_{f}, M\right)^{n}: n \in \mathbb{N}\right)$ converges to $(\mathcal{H}, \mathcal{H})$ (uniformly on compact subsets of $\left.I^{2}\right)$.

Similarly, considering the invariance of the harmonic mean $\mathcal{H}$ with respect to the mean-type mappings involving at least one of the introduced means $G_{f}$ or $H_{f}$ one can determine the explicit formulas for the relevant mean-type mappings ensuring the invariance, the limit of sequence of its iterates, as well as the complementary means.

Corollary 2. Let $I \subset(0, \infty)$ be an interval. Assume that $f: I \rightarrow(0, \infty)$ and $\left.\mathrm{id}\right|_{I}-f$ are strictly increasing. The $\mathcal{H}$-complementary to $A_{f}$ mean $M: I^{2} \rightarrow I$ obtained in Remark 6 is not a generalized weighted geometric mean.

Finally we formulate the following 
Problem 3. Let $I \subset(0, \infty)$ be an interval. Find all functions $f, g, h: I \rightarrow$ $(0, \infty)$ satisfying the equation

$$
G_{g} \circ\left(A_{f}, H_{h}\right)=G_{g},
$$

assuming that $f, g, h$ are, respectively, the generators of generalized weighted arithmetic, geometric and harmonic means.

The solution of this problem in the case when $G_{g}$ is symmetric, i.e. if $G_{g}=\mathcal{G}$, is given in Theorem 1 .

\section{Applications: Invariant Functions}

In this section we deal with a more general question: when a bivariable function (not necessarily a mean) is invariant with respect to the considered mean-type mappings.

Applying the results of the previous section we determine the form of all functions which are invariant with respect to the relevant mean-type mappings and continuous on the diagonal $\Delta:=\{(x, x): x \in I\}$, where $I \subset \mathbb{R}$ is an interval.

Proposition 1. Let $I \subset(0, \infty)$ be an interval and let $f: I \rightarrow(0, \infty)$ and $\frac{\left.\mathrm{id}\right|_{I}}{f}$ be strictly increasing functions. A function $\Phi: I^{2} \rightarrow \mathbb{R}$, continuous on the diagonal $\Delta$, satisfies the functional equation

$$
\Phi\left(\frac{f(x)}{f(y)} y, x+y-\frac{f(x)}{f(y)} y\right)=\Phi(x, y), \quad x, y \in I,
$$

if and only if there is a single-variable continuous function $\varphi: I \rightarrow \mathbb{R}$ such that

$$
\Phi(x, y)=\varphi\left(\frac{x+y}{2}\right), \quad x, y \in I .
$$

Proof. Assume that a continuous on the diagonal $\Delta$ function $\Phi: I^{2} \rightarrow \mathbb{R}$ satisfies Eq. (11), and define the function $\varphi: I \rightarrow \mathbb{R}$ by

$$
\varphi(u):=\Phi(u, u), \quad u \in I .
$$

Notice that Eq. (11) can be written in the form

$$
\Phi\left(G_{f}(x, y), M(x, y)\right)=\Phi(x, y), \quad x, y \in I,
$$

where

$$
G_{f}(x, y)=\frac{f(x)}{f(y)} y \quad \text { and } \quad M(x, y)=x+y-\frac{f(x)}{f(y)} y, \quad x, y \in I .
$$

From (14), by induction, we obtain

$$
\Phi(x, y)=\left(\Phi \circ\left(G_{f}, M\right)^{n}\right)(x, y), \quad x, y \in I, n \in \mathbb{N},
$$


where $\left(G_{f}, M\right)^{n}$ denotes the $n$-th iterates of the mean-type mapping $\left(G_{f}, M\right)$. In view of Remark 5 we get

$$
\lim _{n \rightarrow \infty}\left(G_{f}, M\right)^{n}(x, y)=(\mathcal{A}(x, y), \mathcal{A}(x, y))=\left(\frac{x+y}{2}, \frac{x+y}{2}\right), \quad x, y \in I .
$$

Since the function $\Phi$ is continuous on the diagonal $\Delta$ and

$$
\left(\frac{x+y}{2}, \frac{x+y}{2}\right) \in \Delta, \quad x, y \in I,
$$

it follows from (15) and (13) that, for all $x, y \in I$,

$$
\begin{aligned}
\Phi(x, y) & =\lim _{n \rightarrow \infty}\left(\Phi \circ\left(G_{f}, M\right)^{n}\right)(x, y)=\Phi(\mathcal{A}(x, y), \mathcal{A}(x, y)) \\
& =\Phi\left(\frac{x+y}{2}, \frac{x+y}{2}\right)=\varphi\left(\frac{x+y}{2}\right),
\end{aligned}
$$

which proves (12).

Now, assume that there is a continuous function $\varphi: I \rightarrow \mathbb{R}$ such that (13) holds. Then by Remark 5 the arithmetic mean $\mathcal{A}$ is invariant with respect to the mean-type mapping $\left(G_{f}, M\right)$, and, for all $x, y \in I$,

$$
\begin{aligned}
\Phi(x, y) & =\varphi\left(\frac{x+y}{2}\right)=\varphi \circ \mathcal{A}(x, y)=\varphi \circ\left(\mathcal{A} \circ\left(G_{f}, M\right)\right)(x, y) \\
& =\Phi\left(G_{f}(x, y), M(x, y)\right)=\Phi\left(\frac{f(x)}{f(y)} y, x+y-\frac{f(x)}{f(y)} y\right),
\end{aligned}
$$

which proves that the function $\Phi$ satisfies Eq. (11).

Corollary 3. Let $I \subset(0, \infty)$ be an interval and let $f: I \rightarrow(0, \infty)$ and $\frac{\left.\mathrm{id}\right|_{I}}{f}$ be strictly increasing functions. If a function $\Phi: I^{2} \rightarrow \mathbb{R}$ is a mean and satisfies Eq. (11), then $\Phi=\mathcal{A}$.

Proof. Assume that $\Phi: I^{2} \rightarrow \mathbb{R}$ is a mean satisfying Eq. (11). Since every mean is continuous on the diagonal (see [12]), in view of Proposition 1 there is a single-variable continuous function $\varphi: I \rightarrow \mathbb{R}$ such that

$$
\Phi(x, y)=\varphi\left(\frac{x+y}{2}\right), \quad x, y \in I .
$$

Setting here $y=x$, by the reflexivity of every mean, we get

$$
\varphi(x)=\varphi\left(\frac{x+x}{2}\right)=\Phi(x, x)=x, \quad x \in I,
$$

whence

$$
\Phi(x, y)=\frac{x+y}{2}=\mathcal{A}(x, y), \quad x, y \in I
$$


Since each of the results given below can be proved similarly as Proposition 1 or Corollary 3 we omit their proofs.

Proposition 2. Let $I \subset \mathbb{R}$ be an interval and let $f: I \rightarrow \mathbb{R}$ and $\left.\mathrm{id}\right|_{I}-f$ be strictly increasing functions. A function $\Phi: I^{2} \rightarrow \mathbb{R}$, continuous on the diagonal $\Delta$, satisfies the functional equation

$$
\Phi(f(x)+y-f(y), f(y)+x-f(x))=\Phi(x, y), \quad x, y \in I,
$$

if and only if there is a single-variable continuous function $\varphi: I \rightarrow \mathbb{R}$ such that

$$
\Phi=\varphi \circ \mathcal{A} .
$$

Corollary 4. Let $I \subset \mathbb{R}$ be an interval and let $f: I \rightarrow(0, \infty)$ and $\left.\mathrm{id}\right|_{I}-f$ be strictly increasing functions. If a function $\Phi: I^{2} \rightarrow \mathbb{R}$ is a mean and satisfies Eq. (16), then $\Phi=\mathcal{A}$.

Proposition 3. Let $I \subset(0, \infty)$ be an interval and let $f: I \rightarrow(0, \infty)$ and $\left.\mathrm{id}\right|_{I}-f$ be strictly increasing functions. A function $\Phi: I^{2} \rightarrow \mathbb{R}$, continuous on the diagonal $\Delta$, satisfies the functional equation

$$
\Phi\left(\frac{x y}{f(x)+y-f(y)}, x+y-\frac{x y}{f(x)+y-f(y)}\right)=\Phi(x, y), \quad x, y \in I,
$$

if and only if there is a single-variable continuous function $\varphi: I \rightarrow \mathbb{R}$ such that

$$
\Phi=\varphi \circ \mathcal{A} .
$$

Corollary 5. Let $I \subset(0, \infty)$ be an interval and let $f: I \rightarrow(0, \infty)$ and $\left.\mathrm{id}\right|_{I}-f$ be strictly increasing functions. If a function $\Phi: I^{2} \rightarrow \mathbb{R}$ is a mean and satisfies Eq. (17), then $\Phi=\mathcal{A}$.

Proposition 4. Let $I \subset(0, \infty)$ be an interval and let $f: I \rightarrow(0, \infty)$ and $\left.\mathrm{id}\right|_{I}-f$ be strictly increasing functions. A function $\Phi: I^{2} \rightarrow \mathbb{R}$, continuous on the diagonal $\Delta$, satisfies the functional equation

$$
\Phi\left(f(x)+y-f(y), \frac{x y}{f(x)+y-f(y)}\right)=\Phi(x, y), \quad x, y \in I,
$$

or

$$
\Phi\left(\frac{x y}{f(x)+y-f(y)}, f(x)+y-f(y)\right)=\Phi(x, y), \quad x, y \in I,
$$

if and only if there is a single-variable continuous function $\varphi: I \rightarrow \mathbb{R}$ such that

$$
\Phi=\varphi \circ \mathcal{G}
$$


Corollary 6. Let $I \subset(0, \infty)$ be an interval and let $f: I \rightarrow(0, \infty)$ and $\left.\mathrm{id}\right|_{I}-f$ be strictly increasing functions. If a function $\Phi: I^{2} \rightarrow \mathbb{R}$ is a mean and satisfies Eq. (18) or (19), then $\Phi=\mathcal{G}$.

Proposition 5. Let $I \subset(0, \infty)$ be an interval and let $f: I \rightarrow(0, \infty)$ and $\frac{\left.\mathrm{id}\right|_{I}}{f}$ be strictly increasing functions. A function $\Phi: I^{2} \rightarrow \mathbb{R}$, continuous on the diagonal $\Delta$, satisfies the functional equation

$$
\Phi\left(\frac{f(x)}{f(y)} y, \frac{f(y)}{f(x)} x\right)=\Phi(x, y), \quad x, y \in I,
$$

if and only if there is a single-variable continuous function $\varphi: I \rightarrow \mathbb{R}$ such that

$$
\Phi=\varphi \circ \mathcal{G}
$$

Corollary 7. Let $I \subset(0, \infty)$ be an interval and let $f: I \rightarrow(0, \infty)$ and $\frac{\left.\mathrm{id}\right|_{I}}{f}$ be strictly increasing functions. If a function $\Phi: I^{2} \rightarrow \mathbb{R}$ is a mean and satisfies Eq. (20), then $\Phi=\mathcal{G}$.

Proposition 6. Let $I \subset(0, \infty)$ be an interval and let $f: I \rightarrow(0, \infty)$ and $\left.\mathrm{id}\right|_{I}-f$ be strictly increasing functions. A function $\Phi: I^{2} \rightarrow \mathbb{R}$, continuous on the diagonal $\Delta$, satisfies the functional equation

$$
\Phi\left(f(x)+y-f(y), \frac{x y(f(x)+y-f(y))}{(x+y)(f(x)-f(y))+y^{2}}\right)=\Phi(x, y), \quad x, y \in I,
$$

or

$$
\Phi\left(\frac{x y}{f(x)+y-f(y)}, \frac{y x}{f(y)+x-f(x)}\right)=\Phi(x, y), \quad x, y \in I,
$$

if and only if there is a single-variable continuous function $\varphi: I \rightarrow \mathbb{R}$ such that

$$
\Phi=\varphi \circ \mathcal{H}
$$

Corollary 8. Let $I \subset(0, \infty)$ be an interval and let $f: I \rightarrow(0, \infty)$ and $\left.\mathrm{id}\right|_{I}-f$ be strictly increasing functions. If a function $\Phi: I^{2} \rightarrow \mathbb{R}$ is a mean and satisfies Eq. (21) or (22), then $\Phi=\mathcal{H}$.

Proposition 7. Let $I \subset(0, \infty)$ be an interval and let $f: I \rightarrow(0, \infty)$ and $\frac{\left.\mathrm{id}\right|_{I}}{f}$ be strictly increasing functions. A function $\Phi: I^{2} \rightarrow \mathbb{R}$, continuous on the diagonal $\Delta$, satisfies the functional equation

$$
\Phi\left(\frac{f(x)}{f(y)} y, \frac{x y f(y)}{(x+y) f(x)-x f(y)}\right)=\Phi(x, y), \quad x, y \in I,
$$

if and only if there is a single-variable continuous function $\varphi: I \rightarrow \mathbb{R}$ such that

$$
\Phi=\varphi \circ \mathcal{H}
$$


Corollary 9. Let $I \subset(0, \infty)$ be an interval and let $f: I \rightarrow(0, \infty)$ and $\frac{\left.\mathrm{id}\right|_{I}}{f}$ be strictly increasing functions. If a function $\Phi: I^{2} \rightarrow \mathbb{R}$ is a mean and satisfies Eq. (23), then $\Phi=\mathcal{H}$.

We finish this section with the following

Remark 7 . The condition of the continuity of $\Phi$ on the diagonal $\Delta$ in Propositions 1-7 cannot be omitted. Indeed, for example, the function $\Phi: I^{2} \rightarrow \mathbb{R}$ defined by

$$
\Phi(x, y):=\left\{\begin{array}{l}
0, \text { if }(x, y) \in I^{2} \backslash \Delta, \\
1, \text { if }(x, y) \in \Delta,
\end{array}\right.
$$

satisfies Eqs. (11), (16) and (17), is discontinuous at every point of $\Delta$ and, of course, it is not of the form $\varphi \circ \mathcal{A}$ where $\varphi$ is a single variable function defined on $I$.

Funding The authors have not disclosed any funding.

Data Availability Data sharing not applicable to this article as no datasets were generated or analysed during the current study.

\section{Declarations}

Conflict of interest The authors declare that they have no conflict of interest.

Open Access. This article is licensed under a Creative Commons Attribution 4.0 International License, which permits use, sharing, adaptation, distribution and reproduction in any medium or format, as long as you give appropriate credit to the original author(s) and the source, provide a link to the Creative Commons licence, and indicate if changes were made. The images or other third party material in this article are included in the article's Creative Commons licence, unless indicated otherwise in a credit line to the material. If material is not included in the article's Creative Commons licence and your intended use is not permitted by statutory regulation or exceeds the permitted use, you will need to obtain permission directly from the copyright holder. To view a copy of this licence, visit http://creativecommons.org/ licenses/by $/ 4.0 /$.

\section{References}

[1] Baják, Sz., Páles, Zs.: Invariance equation for generalized quasi-arithmetic means. Aequationes Math. 77, 133-145 (2009)

[2] Borwein, J.M., Borwein, P.B.: Pi and the AGM. Monographies et Études de la Société Mathématique du Canada. Wiley, Toronto (1987)

[3] Bullen, P.S.: Handbook of Means and Their Inequalities. Kluwer Academic Publishers, Dordrecht (2003) 
[4] Daróczy, Z., Páles, Z.: The Matkowski-Sutô problem for weighted quasiarithmetic means. Acta Math. Hung. 100, 237-243 (2003)

[5] Głazowska, D., Jarczyk, W., Matkowski, J.: Arithmetic mean as a linear combination of two quasi-arithmetic means. Publ. Math. Debr. 61, 455-467 (2002)

[6] Jarczyk, J.: Invariance of weighted quasi-arithmetic means with continuous generators. Publ. Math. Debr. 71, 279-294 (2007)

[7] Jarczyk, J., Jarczyk, W.: Invariance of means. Aequationes Math. 92, 801-872 (2018)

[8] Kahlig, P., Matkowski, J.: Generalization of the harmonic weighted mean via Pythagorean invariance identity and application. Ann. Math. Sil. 34, 104-122 (2020)

[9] Matkowski, J.: Invariant and complementary quasi-arithmetic means. Aequationes Math. 57, 87-107 (1999)

[10] Matkowski, J.: Iterations of mean-type mappings and invariant means. Ann. Math. Sil. 13, 211-226 (1999)

[11] Matkowski, J.: Chapter 36: Generalized weighted arithmetic means. In: Rassias, T.M., Brzdek, J. (eds.) Functional Equations in Mathematical Analysis, pp. 563-582. Springer, New York (2012)

[12] Matkowski, J.: Iterations of the mean-type mappings and uniqueness of invariant means. Ann. Univ. Sci. Bp. Sect. Comput. 41, 145-158 (2013)

Dorota Głazowska and Janusz Matkowski

Institute of Mathematics

University of Zielona Góra

Szafrana 4A

65-516 Zielona Góra

Poland

e-mail: D.Glazowska@wmie.uz.zgora.pl;

j.matkowski@wmie.uz.zgora.pl

Received: March 15, 2021.

Accepted: January 18, 2022.

Publisher's Note Springer Nature remains neutral with regard to jurisdictional claims in published maps and institutional affiliations. 